\title{
Nitric Oxide-Releasing Acetylsalicylic Acid Derivative
}

National Cancer Institute

\section{Source}

National Cancer Institute. Nitric Oxide-Releasing Acetylsalicylic Acid Derivative. NCI

Thesaurus. Code C61499.

A nitric oxide (NO) donating derivative of acetylsalicylic acid with anti-inflammatory, analgesic, antipyretic, antithrombotic, gastroprotective and potential antitumor activities. The acetylsalicylic acid derivative moiety of this agent inhibits the activities of cyclooxygenase (COX) I and II, preventing the formation of prostaglandins and thromboxanes. A reduction in prostaglandin synthesis accounts for this agent's antiinflammatory, anti-pyretic and analgesic activities; a reduction in thromboxane A2 synthesis results in an irreversible inhibition of platelet aggregation. NO donation by this agent, after cleavage from the acetylsalicylic acid derivative in vivo, may protect the gastric mucosa against the damaging effects of the aspirin derivative by modulating prostaglandins. In tumor cells, the NO donating moiety may block the cell cycle in the G1 and G2 phases and may induce apoptosis through caspase-mediated mechanisms. 